Case report

\title{
DEMODEX CATI AND FELINE IMMUNODEFICIENCY VIRUS CO-INFECTION IN A CAT
}

\author{
P. T. ILIEV, G. ZHELEV, A. IVANOV \& P. PRELEZOV
}

Department of Veterinary Microbiology, Infectious and Parasitic Diseases, Faculty of Veterinary Medicine, Trakia University, Stara Zagora, Bulgaria

\section{Summary}

Iliev, P. T., G. Zhelev, A. Ivanov \& P. Prelezov, 2019. Demodex cati and feline immunodeficiency virus co-infection in a cat. Bulg. J. Vet. Med., 22, No 2, 237-242.

A case of co-infection due to Demodex cati and feline immunodeficiency virus in a cat is described. A 5 year-old neutered male cat was presented to the Small Animal Clinic at the Faculty of Veterinary Medicine, Trakia University with lethargy, diarrhoea, pyrexia, anorexia, adipsia, skin lesions, intense itching and alopecia. Parasitological, mycological and serological examinations were carried out. Adult $D$. cati mites and antibodies against FIV were detected. A proper therapy with an endectocide, antibiotic and antihistamine drug was prescribed. The improvement of the general condition was observed after a month of treatment. Later, the disease recurred twice and the animal was humanely euthanased for medical reasons and at the owner's request.

Key words: cat, Demodex cati, demodicosis, feline immunodeficiency virus (FIV)

Parasitic skin diseases are an important part of feline dermatology in general. There are several main types of mange in the cat including otodectosis, notoedrosis, cheyletiellosis and demodicosis. Ear otodectosis is the most prevalent among cat population in some Balkan countries such as Greece (Sotiraki et al., 2001), Romania (Cozma et al., 2013) and Albania (Knaus et al., 2013). In contrast, demodicosis is considered a rare disease in cats (Kano et al., 2012) and few cases have been reported. Foley (1995) described feline demodicosis as the least commonly diagnosed disease associated with mites in cat.
The medical records of 1407 cats with dermatologic diagnoses at the Cornell University teaching hospital have revealed the presence of 9 cats infested by demodectic mites (Scott et al., 2012). Cases of this disease have also been reported by Guaguere et al. (2004); Lowenstein et al. (2005); Neel et al. (2007); Beale (2012); Kano et al. (2012); Cozma et al. (2013); Silbermayr et al. (2013); Bizikova (2014). In contrast, the feline roundworm Toxocara cati is one of the most prevalent intestinal helminths in cats worldwide.

Feline demodicosis is caused by $\mathrm{De}$ modex cati, Demodex gatoi and an un- 
named species. Demodex gatoi inhabits the superficial skin layer and, in contrast to the others, it is highly contagious and easy transmittable (Silbermayr et al., 2013). Deep follicular-dwelling D. cati is considered to be a normal commensal of cat skin but the normal habitat of D. gatoi is unknown (Medleau \& Hnilica, 2006). Demodicosis due to $D$. cati is usualy triggered by an underlying systemic disease including feline immunodeficiency virus (FIV) infection (Lowenstein et al., 2005). FIV is a retrovirus and appears to infect both CD4+ and CD8+ lymphocytes as well as macrophages (Hirsh \& Zee, 1999). It is followed by progressive immune suppression and increased host susceptibility to opportunistic infection (Teixeira et al., 2012). The virus is excreted with the saliva and the most important route of transmission is probably through bites, therefore male cats with outdoor access, which are most likely to fight, are most frequently infected with FIV (Hirsh \& Zee, 1999).

Demodicosis due to $D$. cati is manifested in two clinical forms. Localised demodicosis is self-limiting and characterised by presence of variably pruritic lesions comprising alopecia, patchy erythema, scaling and crusting (Scott et al., 2001). Lesions usually involve the eyelids, the skin around the eyes, affect the head and the neck (Medleau \& Hnilica, 2006). Demodex cati may also be the cause of a ceruminous otitis externa (Beale, 2012). Generalised disease is rare and lesions are found primarily on the head but could be identified on the neck, trunk and limbs (Scott et al., 2001).

Case presentation. The case refers to a 5 year-old neutered male domestic cat, weighing $4 \mathrm{~kg}$, presented to the Small Animal Clinic because of lethargy, diarrhoea, anorexia, adipsia, skin lesions, in- tense itching and self-induced alopecia which have progressively worsened over about 1-year period. The cat was not vaccinated and lived indoor but also had access to outside areas. Cat was previously treated locally with neomycin and prednisolone (Cortizeme ${ }^{\circledR}$,Virbac), subcutaneously with dexamethasone (Dexafort ${ }^{\circledR}$, Intervet) and a unknown injectable antibiotic in another veterinary clinic but without satisfactory outcome.

The physical examination was made by following the routine protocol. An inspection and body temperature measurement were also carried out. For parasitological examination, swab samples from both ear canals and deep and superficial scraping from the lesions were mixed with lactic acid, mounted on glass slides and coverslipped for microscopic observation. Demodectic mites were identified by morphometrical characteristics of adult forms described by Lowenstein et al. (2005). The faecal sample was processed by standard salt flotation technique. It was also assayed for presence of protozoan species by immunochromatographic rapid test (RIDA ${ }^{\circledR}$ QUICK Cryptosporidium/ Giardia/Entamoeba Combi test, R-Biopharm) according to the manufacturer's instructions.

Hairs and crusts from affected areas were cultivated aerobically on Mycosel agar (BD, USA), at temperature of $27{ }^{\circ} \mathrm{C}$ for 3 weeks.

Blood serum was assayed for presence of FIV antibodies and feline leukaemia virus (FeLV) antigen by Anigen Rapid FIV Ab/FeLV Ag Test (BIONOTE, Inc.) and Toxoplasma gondii antigen by Quicking Feline Toxoplasma gondii antigen test - Toxo Ag (Quicking Biotech Co., Ltd.), both according to the manufacturer's instructions. 
Doramectin (Dectomax ${ }^{\circledR}$, Zoetis) at a dose of $300 \mu \mathrm{g} / \mathrm{kg}$ was subcutaneously applied once weekly for 8 weeks. A systemic antimicrobial therapy was performed orally by amoxicillin and clavulanic acid tablets (Synulox ${ }^{\circledR}$, Zoetis) at a dose of $12.5 \mathrm{mg} / \mathrm{kg}$ divided into two doses per day for 3 weeks. Cetirizine $\left(Z_{y r t e c}{ }^{\circledR}\right.$, UCB Pharma S.A) tablets were also orally administred at dose of $2.5 \mathrm{mg}$ once a day. A control examination was performed 1 month after treatment.

Physical examination revealed the presence of lesions consisting of alopecia with scaling, crusting and excoriations on the face (Fig. 1) and the back (Fig. 2). A mild waxy debris was present in both ear canals. The cat was febrile and the rectal temperature was $39.8{ }^{\circ} \mathrm{C}$. The swab specimen was negative for $O$. cynotis. No Cheyletiella and Notoedres mites were discovered after deep and superficial skin scraping but adult $D$. cati mites were present (Fig. 3). Their body length was about $200 \mu \mathrm{m}$ (Fig. 4). Faecal examination revealed the presence of $T$. cati eggs. No positive reactions of antigen test for Giardia, Cryptosporidium and Entamoeba we-

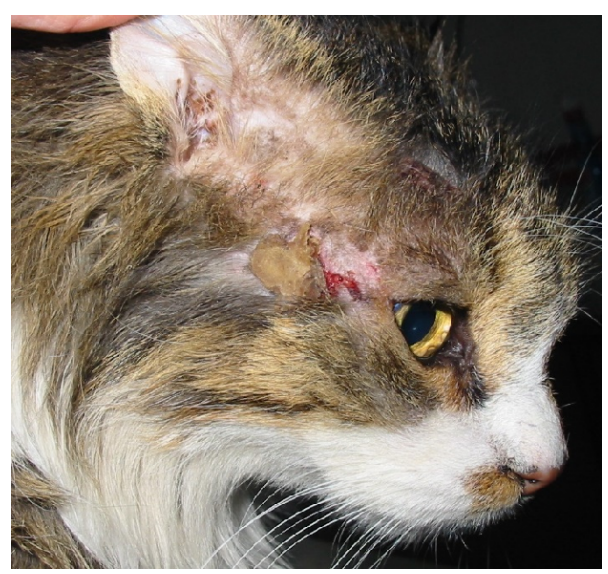

Fig. 1. Crusts and excoriations on the face of the patient. re recorded. The mycological observation was also negative for dermatophytes. Blood serum reacted positively for FIV antibodies and negatively for FeLV and Toxolasma antigens.

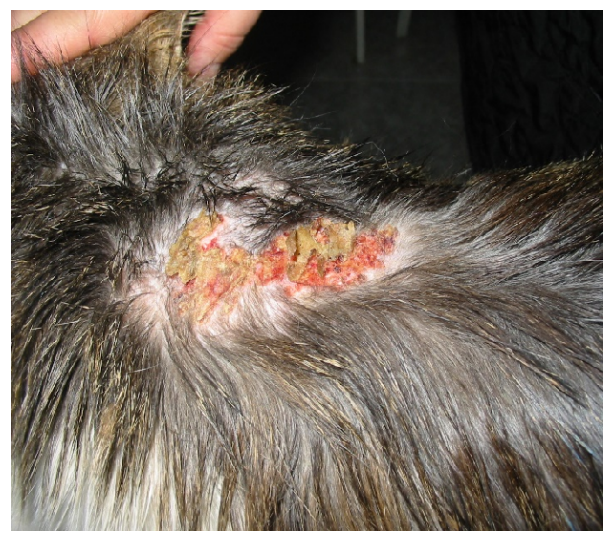

Fig. 2. A large skin lesion on the back.

The skin scraping performed 1 month after treatment with Dectomax ${ }^{\circledR}$, Synulox ${ }^{\circledR}$ and Zyrtec ${ }^{\circledR}$ showed no presence of mites and improved general condition. Faecal flotation also affirmed the lack of $T$. cati eggs. A year later, the disease recurred but the clinical manifestation was more severe than the previous episode. The same treatment was prescribed and all clinical signs resolved again. The new recurrence of signs appeared 6 months later. The general condition has progressively worsened. The cat was increasingly lethargic and anorectic, with profuse diarrhoea, weight loss, inflammation of the gingivas, respiratory symptoms and behavioural abnormalities. No treatment was applied and the cat was humanely euthanised for medical reasons and at the owner's request.

Feline demodicosis caused by $D$. cati is a rare infection in cats. D. cati is considered to be a cat skin commensal (Medleau \& Hnilica, 2006). Demodicosis due to $D$. cati is usualy triggered by an underlying systemic disease such as diabe- 


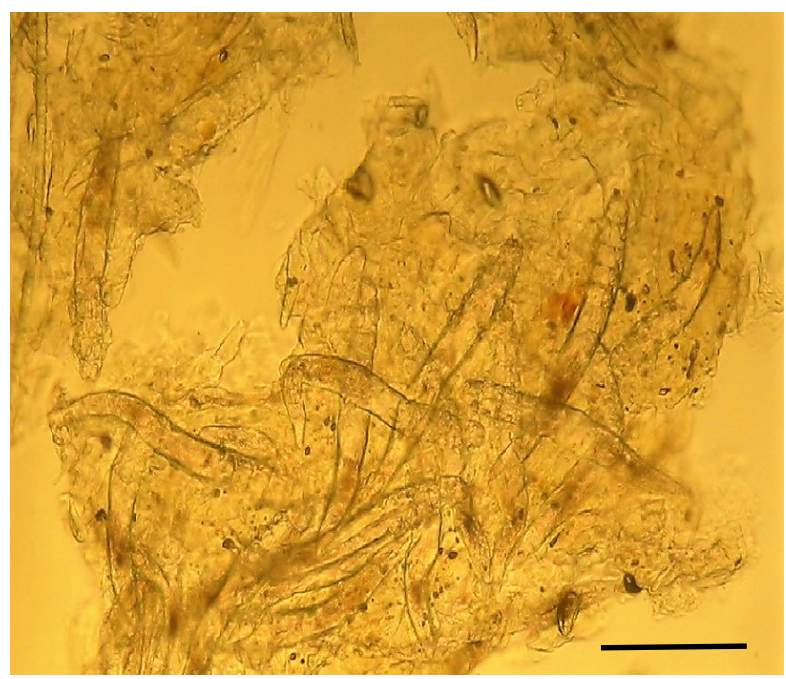

Fig. 3. Demodex cati after deep skin scraping (bar=100 $\mu \mathrm{m})$.

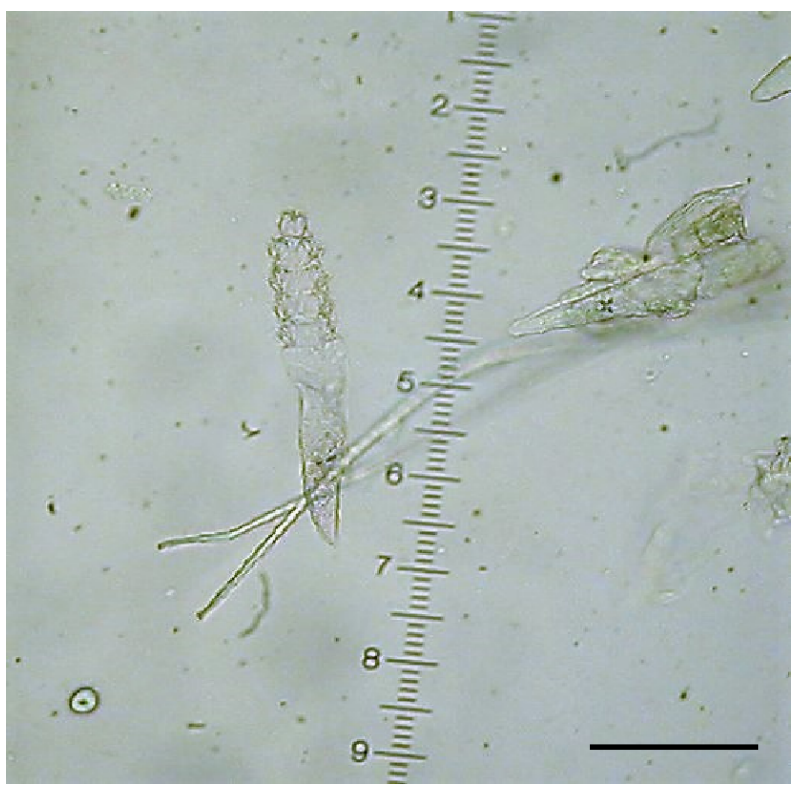

Fig. 4. Adult of Demodex cati (bar=100 $\mu \mathrm{m})$.

tes mellitus, FIV and FeLV infections, hyperadrenocorticism, systemic lupus erythematosus, squamous cell carcinoma in situ, chronic upper respiratory tract infection and toxoplasmosis (Lowenstein et al., 2005). In our case, the cat turned out to be positive for FIV infection. FIV is followed by progressive immune suppression and increased host susceptibility to opportunistic infection (Teixeira et al., 2012). The virus is excreted with the saliva and the most important route of 
transmission is probably through bites (Hirsh \& Zee, 1999). In our case the cat resided indoor but had also an access to outside areas. In addition, vaccination has not been performed before. According to the owner, the first clinical signs had appeared 1 year before and persisted with several remission periods. In our opinion, the cat was infected several years ago. It is very likely because the infection develops very slowly (Hartmann, 2012). Moreover, the previous treatment has been inadequate. Prolonged treatment with prednisolone and dexamethasone is contraindicated in viral infections because of their anti-inflammatory effect but they were probably applied to stop the pruritus. Furthermore, no acaricide drug has been prescribed. Dectomax ${ }^{\circledR}$ was included in the therapy (active against both mites and helminths). Dexamethasone and Cortizem $^{\circledR}$ were replaced with a second generation antihistamine Zyrtec ${ }^{\circledR}$, which also has an antipruritic effect but does not induce immunosuppression. In our case the cat was itchy, which is not a typical sign of $D$. cati infestation. According to Lowenstein et al. (2005) the itching in this disease varies from absent to extreme, and is probably due to secondary skin infection by opportunistic agents. The lesions responded well to the treatment with Dectomax $^{\circledR}$ and the control scraping was negative at the end of therapy. Later, we observed two acute periods of the disease and the FIV syndrome was completely manifested. This clinical manifestation was also observed by Kanzaki \& Looney (2004) who reported that during the progression of FIV infection to the terminal AIDS-like phase, the cats develop AIDS related complex symptoms, opportunistic infections, neoplasia and neurological abnormalities which may eventually lead to death. Similarly to our case, Mircean et al. (2013) observed a multiple-infection with D. cati, FIV, Toxoplasma gondii and Microsporum canis. The authors reported that as a consequence of poor clinical evaluation, the owner choosed euthanasia two weeks later. In the terminal stage of our case, the cat was increasingly lethargic and anorectic, lost weight, had inflammation of the gingiva, respiratory symptoms and behavioural abnormalities. Treatment was not applied and the cat was humanely euthanised.

In conclusion, we recommend timely vaccination of all cats with age-appropriate vaccines and avoiding direct contacts with stray cats. In addition, all cats diagnosed with $D$. cati should be additionally tested for feline immunosuppressive viral infections such as FIV and FeLV.

\section{REFERENCES}

Beale, K., 2012. Feline demodicosis. A consideration in the itchy or overgrooming cat. Journal of Feline Medicine and Surgery, 14, 209-213.

Bizikova, P., 2014. Localized demodicosis due to Demodex cati on the muzzle of two cats treated with inhalant glucocorticoids. Veterinary Dermatology, 25, 222-225.

Cozma, V., M. Dumitrache, M. Mircean, A. Gyorke \& V. Mircean, 2013. Prevalence of ectoparasites in a household cat (Felis catus) populations from Transylvania, Romania. In: Proceedings of the $12^{\text {th }}$ International Symposium on Ectoparasites of Pets, Munich, pp. 63-64.

Foley, R. H., 1995. Feline demodicosis. Compendium on Continuing Education for the Practising Veterinarian, 17, 481-487.

Guaguere, E., A. Muller \& F. DegorceRubiales, 2004. Feline demodicosis: A retrospective study of 12 cases. Veterinary Dermatology, 15, 20-40.

Hartmann, K., Clinical aspects of feline retroviruses: A review. Viruses, 4, 2684-2710. 
Hirsh, D. \& Y. Zee, 1999. Veterinary Microbiology. Blackwell Science, Inc.

Kano, R., A. Hyuga, J. Matsumoto, S. Nogami, S. Nemoto, A. Hasegawa \& H. Kamata, 2012. Feline demodicosis caused by an unnamed species. Research in Veterinary Science, 92, 257-258.

Kanzaki, L. \& D. Looney, 2004. Feline immunodeficiency virus: A concise review. Frontiers in Bioscience, 9, 370-377.

Knaus, M., E. Shukullari, D. Rapti, R. Postoli, D. Xhaxhiu, M. Visser, R. Winter \& S. Rehbein, 2013. Ectoparasite fauna of cats from Tirana, Albania. In: Proceedings of the $12^{\text {th }}$ International Symposium on Ectoparasites of Pets, Munich, pp. 34.

Lowenstein, C., W. Beck, K. Bessmann \& R. S. Mueller, 2005. Feline demodicosis caused by concurrent infestation with $\mathrm{De}$ modex cati and an unnamed species of mite. The Veterinary Record, 157, 290292.

Medleau, L. \& K. A. Hnilica, 2006. Small Animal Dermatology: A Color Atlas and Therapeutic Guide, $2^{\text {nd }}$ edn, Elsevier Inc., St. Louis, Missouri.

Mircean, V., M. Dumitrache, M. Mircean, A. Gyorke \& V. Cozma, 2013. Concurrent demodicosis and dermatophytosis in a cat with feline immunodeficiency virus (FIV) - case report. In: Proceedings of the $12^{\text {th }}$ International Symposium on Ectoparasites of Pets, Munich, pp. 62.

Neel, J., J. Tarigo, K. Tater \& C. Grindem, 2007. Deep and superficial skin scrapings from a feline immunodeficiency viruspositive cat. Veterinary Clinical Pathology, 36, 101-104.
Scott, D., W. Miller, C. Griffin, 2001. Muller $\&$ Kirk's Small Animal Dermatology, $6^{\text {th }}$ edn, Elsevier, Philadelphia, PA.

Scott, D., W. Miller \& H. Erb, 2012. Feline dermatology at Cornell University: 1407 cases (1988-2003). Journal of Feline Medicine and Surgery, 15, 307-316.

Silbermayr, K., A. Joachim, B. Lischauer, N. Sastre, L. Ferrer \& C. Horvath-Ungerbock, 2013. The detection and treatment of Demodex gatoi in an Austrian Cornish rex cat. In: Proceedings of the $12^{\text {th }}$ International Symposium on Ectoparasites of Pets, Munich, pp. 53.

Sotiraki, S., A. Koutinas, L. Leontides, K. Adamama-Moraitou \& C. Himonas, 2001. Factors affecting the frequency of ear canal and face infestation by Otodectes cynotis in the cat. Veterinary Parasitology, 96, 309-315.

Teixeira, B., M. Hagiwara, J. Cruz \& M. Hosie, 2012. Feline immunodeficiency virus in South America. Viruses, 4, 383396.

Paper received 13.02.2017; accepted for publication 07.04.2017

\section{Correspondence:}

Petar T. Iliev

Department of Veterinary Microbiology, Infectious and Parasitic Diseases, Faculty of Veterinary medicine, Trakia University, 6000 Stara Zagora, Bulgaria tel: +35942 699587 e-mail: petyo_todorow@abv.bg 